Trocknen bei $133-134^{0}$ schmolzen und auch in dem sonstigen Verhalten durchaus mit $\mathrm{Harns}$ t of $\mathrm{f}$ übereinstimmten.

Die Hauptmenge des aus Calciumcyanamid erhaltenen Rohharnstoffes wurde durch Umkrystallisieren aus Alkohol gereinigt, wobei ein beträchtlicher Teil des Trockenrückstandes ungelöst blieb. Immerhin wurden aus $50 \mathrm{~g}$ rohem Calciumcyanamid $12,5 \mathrm{~g}$ reiner Harnstoff erhalten.

Aus diesen Versuchen geht hervor, daß das C y a n a mid durch Wasser, wenn auch nur in sehr geringem Umfange, direkt in $\mathrm{H}$ a r n s t of $f$ verwandelt wird, und zwar sowohl bei gewöhnlicher Temperatur (in ätherischer Lösung), als auch beim Verdampfen. Wesentlich größer ist die Menge des durch direkte Wasseraufnahme gebildeten Harnstoffes, wenn dem Wasser vor dem Eindampfen Wasserstoffsuperoxydlösung zugesetzt wird. In ähnlicher Weise vollzieht sich die Umwandlung von Cyanamid in Harnstoff nicht nur bei Gegenwart von Essigsäure (siehe vorstehende Abhandlung), sondern auch von Oxalsäure und vermutlich auch noch von anderen stärkeren organischen Säuren.

Mitteilungen aus dem pharmazeutisch-chemischen Institut der Universitat Marburg.

\title{
260. Ueber die Bestimmung des metallischen Eisens im Ferrum reductum.
}

Von Dr. A u gust Eberhard.

(Eingegangen den 6. VIII. 1917.)

Die Bestimmung des Gehaltes an metallischem Eisen im Ferrum reductum hat in den letzten $30 \mathrm{Jahren}$ wiederholt den Gegenstand eingehender Untersuchungen gebildet, ohne daß es jedoch bisher gelungen ist, eine einfache, allgemein anwendbare Prüfungsvorschrift hierfür einwandfrei aufzustellen. Es mußte daher von großem Interesse sein, da $B$ L. W. W i n $\mathbf{k}$ l e $\mathbf{r}^{\mathbf{1}}$ ) vor kurzem Mitteilung über eine sehr einfache, bisher jedoch ziemlich unbekannt gebliebene Methode zur Bestimmung des Gehaltes an metallischem Eisen im Ferrum reductum machte.

Nach $W$ i n k le r läßt sich der Gehalt an metallischem Eisen in dem mit Wasserstoff reduzierten Eisen einfach dadurch ermitteln,

1) Ztschr. f. angew. Chem. 1917, 64. 
daß man eine Probe davon an der Luft glüht und die hierdurch eintretende Gewichtszunahme feststellt. Die betreffenden Versuche zeigten, daß 100 Gewichtsteile reduziertes Eisen bei anhaltendem. Glühen an der Luft sich zu 142,0 Gewichtsteilen Oxyd umwandeln, so daß sich hierbei im wesentlichen Ferrioxyd bildet. Wird nach diesem Verfahren genau $1 \mathrm{~g}$ Ferrum hydrogenio reduotum angewendet, dasselbe 15-20 Minuten lang mit einer Bunsenflamme. heftig geglüht, der Rückstand nach dem Erkalten gewogen und dann zur Sicherheit noch ein zweites Mal geglüht, so kann der Prozentgehalt an metallischem Eisen direkt einer von $\mathrm{W}$ in $\mathbf{k} \mathrm{l}$ er beigefügten Tabelle entnommen werden. Für jede weitere, in dieser Tabelle nicht enthaltene Gewichtæzunahme kann dann für je $1 \mathrm{mg}$ $0,25 \%$ metallisches Eisen in Rechnung gestellt werden, und sollen auf diese Weise bis auf $0,5 \%$ genaue Werte erhalten werden.

Dieses Verfahren ist von $\mathrm{W}$ in $\mathbf{k l e ~} \mathbf{r}^{\mathbf{1}}$ ) bereits 1892 vorgeschlagen worden und hat dasselbe später in der ungarischen Pharmakopöe Aufnahme gefunden. Es muß daher überraschen, daß dieses, durch seine Einfachheit ausgezeichnete Untersuchungsverfahren sich bisher nicht als Bestimmungsmethode einzubürgern vermochte. Eine Nachprüfung dieser Methode schien daher um so mehr von Interesse zu sein, als W i n k l e r (l. c.) vor kurzem erneut auf dieselbe aufmerksam gemacht hat.

Bei der Nachprüfung dieser W inkler'schen Eisenbestimmungsmethode machte ich jedoch Beobachtungen, welche Zweifel an der allgemeinen Anwendbarkeit derselben aufkommen ließen.

Zunächst erwies sich das von $\mathrm{W}$ in $\mathrm{kle} \mathrm{r}$ angegebene zweimalige, 15-20 Minuten lange Glühen des Eisens bei allen von mir ausgeführten Versuchen zur vollständigen Oxydation desselben als unzureichend. Je nach der Art des angewendeten Ferrum reductum mußte das Glühen desselben 5-10mal wiederholt werden, ehe ein einigermaßen konstantes Gewicht erzielt werden konnte, gleichgültig, ob dabei gewöhnliche Bunsenbrenner oder Pilzbrenner als Heizquelle benutzt wurden. Das Fortschreiten des Oxydationsvorganges konnte hierbei an der Farbenveränderung des wieder erkalteten Glührückstandes beobachtet werden Erst dann, wenn die schwarze Färbung des Oxydationsproduktes annähernd vollständig in Braun übergegangen war, konnte die Reaktion als beendet angesehen werden. Dieser Uebergang wurde aber nie nach ein- oder zweimaligem Glühen erreicht, vielmehr zeigte dann der Tiegel- bzw. Schaleninhalt stets noch eine schwarze, mehr dem Eisen-

1) Gyógyszerészi Közlöny 8., 258 u. 313 (1892). 
oxyduloxyd entsprechende Färbung. Auch die Gewichtszunahme des zweimal bei Luftzutritt stark geglühten Eisens entsprach durchaus nicht derjenigen, welche nach dem Gehalt an metallischem Eisen hätte bei vollständiger Oxydation eintreten müssen. Dieses Verhalten dürfte wohl dadurch bedingt sein, daß bei der zunächst unter Aufglimmen rasch erfolgenden Oxydation des Eisens ein Zusammenbacken der ganzen Masse durch das schmelzbare Eisenoxyduloxyd stattfindet und infolgedessen, je nachdem das Eisen in dünnerer oder dickerer Schicht ausgebreitet ist, eine Bildung von mehr oder minder starken Krusten hervorgerufen wird. Hierdurch wird die weitere Einwirkung des Sauerstoffes auf die tieferliegenden Schichten verhindert oder doch sehr erschwert, so daß das Eisenoxyduloxyd entweder nur oberflächlich oder doch nur ganz allmählich durch mehrfach wiederholtes Glühen in Eisenoxyd übergeführt wird. Ferner wurde beobachtet, daß in dickeren Schichten oder dann, wenn die Krusten sich teilweise von dem Boden der Schale loslösten (wie bei Versuch III), infolge des dem Eisenoxyduloxyd eigenen geringen Wärmeleitungsvermögens überhaupt keine gleichmäßige Braunfärbung des geglühten Materials und damit auch keine Gewichtskonstanz zu erzielen war.

Die Nachprüfung des $W$ inkle r'schen Verfahrens wurde. zunächst in einem breiten, offenen Tiegel vorgenommen. Genau $1 \mathrm{~g}$ Ferrum reductum wurde jedesmal 20 Minuten lang stark geglüht, nach halbstündigem Erkalten im Exsikkator die Gewichtszunahme festgestellt, und daraus der Prozentgehalt an metallischem Eisen berechnet.

Versuch I.

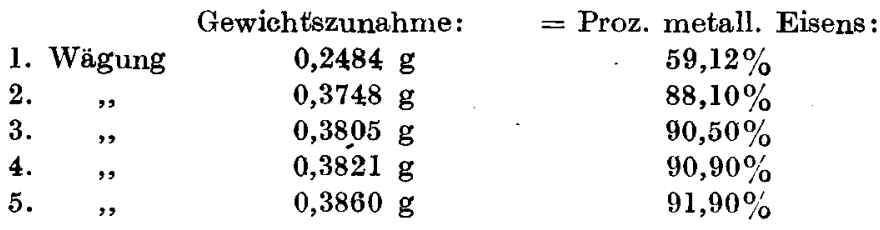

Da das zu diesem Versuch angewendete Ferrum reductum nach der direkten Bestimmung 93,74-94,53\% metallisches Eisen enthielt (siehe S. 368), so war dieser Wert auch nach fünfmaligem starken Glühen noch nicht erreicht worden.

$\mathrm{Da}$ das Eisen bei diesem Versuch zu einem festen Kuchen zusammengebacken war, und diesem Umstand vielleicht die langsame Gewichtszunahme zugeschrieben werden konnte, so wurde das Verfahren unter Anwendung einer größeren Platinschale mit flachem Boden (Weinschale) wiederholt und hierbei gleichzeitig für eine 
möglichst gleichmäßige Verteilung des Eisens Sorge getragen. Indessen erwies sich auch in diesem Falle ein mehrfach wiederholtes Glühen als erforderlich. Das Glühen wurde jedesmal auf 20 Minuten ausgedehnt; zur 'Anwendung gelangte dasselbe Präparat wie bei Versuch $I$.

Versuch II.

Gewichtszunahme eines Grammes = Proz. metall. Eisens:

Ferrum reductum:

$\begin{array}{lll}\text { 1. Wägung } & 0,2472 \mathrm{~g} & \mathbf{5 8 , 8 3} \% \\ \text { 2. }, & 0,3039 \mathrm{~g} & \mathbf{7 2 , 3 0 \%} \\ \text { 3. } \quad, & 0,3190 \mathrm{~g} & \mathbf{7 6 , 0 5 \%} \\ \text { 4. } & 0,3536 \mathrm{~g} & 84,30 \% \\ \text { 5. } & 0,3697 \mathrm{~g} & 88,10 \% \\ \text { 6. } & 0,3709 \mathrm{~g} & \mathbf{8 8 , 3 3 \%}\end{array}$

Eigentümlicherweise ergab also dieser Versuch mit fein? verteiltem Material trotz der wesentlich größeren Oberfläche einen um fast 3,6\% niedrigeren Wer亡 als der frühere.

Zur Kontrolle wurde schließlich noch $1 \mathrm{~g}$ einer geringwertigeren Eisenprobe unter denselben Versuchsbedingungen erhitzt. Indessen war bei diesem Versuch überhaupt keine Gewichtskonstanz zu erzielen, da sich einzelne Krusten von dem Schalenboden loslösten und trotz stärksten Glühens nicht in Eisenoxyd übergeführt werden konnten. Da auch bei dem zeñnten, über eine halbe Stunde ausgedehnten Erhitzen eine weitere Gewichtszunahme eintrat, und das geglühte Material noch immer inhomogen war, wurde der Versuch als erfolglos abgebrochen. Die nach zehnmaligem Glühen erzielte Gewichtszunahme entsprach noch keineswegs dem in Wirklichkeit vorhandenen Gehalt an metallischem Eisen (im Mittel 86,03\%).

Versuch III.

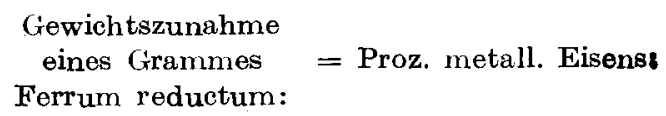

\begin{tabular}{|c|c|c|}
\hline 1. Wägung & $0,1000 \mathrm{~g}$ & $23,80 \%$ \\
\hline 2. & $0,1292 \mathrm{~g}$ & $30,75 \%$ \\
\hline 3. & $0,1861 \mathrm{~g}$ & $44,29 \%$ \\
\hline 4. & $0,2052 \mathrm{~g}$ & $48,84 \%$ \\
\hline 5. & $0,2302 \mathrm{~g}$ & 54,790 \\
\hline 6. & $0,2387 \mathrm{~g}$ & $56,81 \%$ \\
\hline 7. & $0,2434 \mathrm{~g}$ & $57,93^{\circ}$ \\
\hline 8. & $0,2480 \mathrm{~g}$ & 59,02 \\
\hline 9. & $0,2559 \mathrm{~g}$ & 60,90 \\
\hline 10. & $0,2737 \mathrm{~g}$ & $65,14 \%$ \\
\hline
\end{tabular}


Da derartig niedrige Werte in der $W$ in $\mathbf{k}$ l e r'schen Tabelle nicht aufgeführt sind, mußte der Prozentgehalt an metallischem Fisen unter Zugrundelegung der aus den Tabellenwerten entnom menen mittleren Gewichtszunahme berechnet werden. Jeder Gewichtserhöhung um $1 \mathrm{mg}$ entsprechen hiernach 0,238\% metallischen Eisens.

Abgesehen von dem Umstand, daß das W ink ler'sche Verfahren der Eisenbestimmung durch die erforderliche mehrfache Wiederholung des Glühens sehr viel Zeit beansprucht, ferner. Leuchtgas nicht überall zur Verfügung steht, und mit Spiritusbrennern die nötigen hohen Temperaturen nicht $z u$ erzielen sind, lassen obige Ergebnisse die W in k le r'sche Methode für die Bestimmung des metallischen Eisens im Ferrum reductum, wenigstens für das gegenwärtig im Handel befindliche Präparat, wohl kaum als geeignet erscheinen.

Es würde dies nur der Fall sein, wenn es bei der Ausführung dieser Bestimmung möglich wäre, den Punkt scharf zu beobachten, bei welchem die Gesamtmenge des metallischen Eisens zu Eisenoxyd oxydiert ist, das a priori vorhandene Eisenoxyduloxyd jedoch noch nicht verändert wird. Dies ist jedoch nicht möglich, da durch das intermediär gebildete, schmelzende Eisenoxyduloxyd leicht ein Zusammenbacken der geglühten Masse eintritt. Wird dann das Glühen bis zur Gewichtskonstanz fortgesetzt, so erleidet nicht nur dieses, sondern auch das ursprünglich vorhanden gewesene Eisenoxyduloxyd eine Umwandlung in Eisenoxyd, durch welche dann das schließlich bei Gewichtskonstanz erzielte Resultat zu hoch ausfallen muß, vorausgesetzt, daß wirklich eine vollständige Oxydation des gesamten Materials stattfindet.

Welche Gewichtszunahme das reine Eisenoxyduloxyd, welches als reiner, gut krystallisierter Magneteisenstein gepulvert zur Anwendung gelangte, bei jedesmaligem 20 Minuten währendem Glühen bei Luftzutritt erfährt, geht aus den nachstehenden Daten hervor

Gewichtszunahme von $0,6568 \mathrm{~g}$ Magneteisenstein = Prozente:

$\begin{array}{ccc}\text { 1. Wägung } & 0,0084 \mathrm{~g} & 1,28 \% \\ 2 . \quad, & 0,0096 \mathrm{~g} & 1,46 \% \\ 3 . \quad, & 0,0109 \mathrm{~g} & 1,66 \% \\ 4 . \quad ., & 0,0119 \mathrm{~g} & 1,81 \% \\ 5 . \quad ", & 0,0124 \mathrm{~g} & 1,89 \% \\ 6 . \quad, & 0,0134 \mathrm{~g} & 2,04 \% \\ 7 . \quad, & 0,0144 \mathrm{~g} & 2,19 \% \\ 8 . & & \\ 9 \\ 10 .\end{array}$


Wäre das Eisenoxyduloxyd vollständig in Eisenoxyd übergegangen, so hätte die Gewichtszunahme $3,45 \%$ betragen müssen. Wenn dieser theoretische Wert in praxi nicht erreicht wurde, so kann dieser Umstand nur durch die teilweise gröbere Beschaffenheit des angewandten Pulvers bedingt sein, da es kaum gelingt, mit einfachen Hilfsmitteln eine kleine Menge eines Materials restlos in feinstes Pulver zu verwandeln.

Das negative Resultat, welches bei diesen Versuchen erzielt worden war, gab mir Veranlassung zu einer weiteren Beschäftigung mit diesem, in allen Pharmakopöen aufgeführten Präparat, dessen Prüfung, wie bereits erwähnt, schon wiederholt den Gegenstand eingehender Untersuchungen gebildet hat.

A. Mar qua $r \mathbf{d}^{1}$ ) hat bereits darauf hingewiesen, daß die Methode, nach welcher das Eisen im Ferrum reductum bestimmt werden soll, nicht allein mit jeder neuen Auflage des Deutschen Arzneibuches, sondern auch mit denen der ausländischen Pharmakopöen eine Aenderung erfahren hat. Diese Bestimmungsmethoden weichen derartig voneinander ab, daß man bei der Untersuchung desselben Präparates nach denselben kaum bei zweien dieser Methoden übereinstimmende Resultate erhält.

Der häufige Wechsel in den Gehaltsbestimmungsmethoden des Ferrum reductum scheint indessen nur zum Teil durch die Erkenntnis der größeren oder geringeren Zuverlässigkeit der Prüfungsvorschriften selbst bedingt zu sein, sondern auch durch die mit der Zeit abgeänderten Darstellungsverfahren und die hierdurch gegenüber früheren Präparaten unterschiedlichen Eigenschaften des Ferrum reductum.

Schon die Namensänderung dieses Arzneimittels aus „Ferrum hydrogenio reductum" in „Ferrum reductum" läßt darauf schließen, daß in der Neuzeit nicht mehr ausschließlich reiner Wassersioff zur Reduktion dient, sondern daß auch technische, wasserstoffhaltige Gase zur Gewinnung dieses Arzneistoffes Verwendung finden. Während das 1. Deutsche Arzneibuch als Untertitel des Ferrum reductum die präzisere Bezeichnung Ferrum hydrogenio reductum aufführt, ist letztere Benennung bereits von der 2. Ausgabe an unterblieben. Die Annahme, daß diese Aenderung der Bezeichnung dadurch bedingt worden sei, daß zur Darstellung des Ferrum reductum kein reines Wasserstoffgas mehr Verwendung findet, schien durch die Beobachtung bestätigt zu werden, daß die bei der Bestimmung des Gesamteisengehaltes ungelöst bleibenden

1) Chem.-Ztg. 1901, 744. 
Flocken tiefschwarz gefärbt waren, soda $B$ es den Anschein gewann, es sei in dem Rückstand neben Kieselsäure auch Kohle enthalten. Diese Schwarzfärbung des Rückstandes konnte jedoch durch mehrtägiges Erhitzen mit wiederholt erneuerter Salzsäure von 12,5\% auf dem Wasserbad allmählich zum Verschwinden gebracht werden, so daß schließlich nur noch rein weiße Kieselsäureflocken übrig blieben. Demnach ist das Vorhandensein von Kohle in dem in Säure unlöslichen Rückstand des Ferrum reductum, welches einen direkten Anhalt für die Verwendung von kohlenoxydhaltigen Reduktionsmitteln bei der Darstellung desselben gegeben haben würde, wohl ausgeschlossen. Immerhin zeigt dieses Verhalten, daß die jetzigen Handelspräparate von Ferrum reductum Eisenverbindungen enthalten, die selbst in der Wärme in Salzsäure sehr schwer löslich sind.

Jedenfalls ist die bei der Darstellung des Präparates angewandte Temperatur von Einfluß auf die Eigenschaften des Endproduktes. Schon 1897 hat E. S c h m id $\dot{t}^{1}$ ) darauf hingewiesen, daß durch die Anwendung hoher Wärmegrade bei der Reduktion die Beschaffenheit des Präparates insofern leidet, als dadurch ein wesentlich kompakteres und infolgedessen schwerer lösliches Pulver erhalten wird als nach der früher üblichen reinen Wasserstoffreduktion bei niedrigerer Temperatur. Unter diesen Umständen kann es nicht überraschen, daß die jetzigen Handelspräparate im Vergleich mit den früher dargestellten Produkten in den Löslichkeitsverhältnissen. wesentliche Unterschiede zeigen, so daß Untersuchungsmethoden, die früher einwandfreie Resultate lieferten, den veränderten Verhältnissen jetzt nicht mehr gerecht werden und daher nur noch mit Abänderungen brauchbar sind. Auch hierdurch dürfte zum Teil der häufige Wechsel in den von den Arzneibüchern vorgeschriebenen Bestimmungsmethoden und die damit in Verbindung stehenden Verbesserungsvorschläge derselben mit bedingt sein.

Von den Arzneibüchern der verschiedenen Staaten schreiben nur das deutsche und das holländische die Bestimmung des Gesamteisengehaltes vor, während die Pharmakopöen aller anderen Länder die Frmittelung des Gehaltes an metallischem Eisen verlangen, soweit sie sich nicht - wie das spanische Arzneibuch - mit der qualitativen Prüfung begnügen.

Die Zahl der in der Praxis zur Bestimmung des metallischen Eisens im Ferrum reductum angewendeten Verfahren ist dem Prinzip nach zwar nur beschränkt, jedoch weichen dieselben in den Einzelheiten ihrer Ausführung nicht unerheblich voneinander

1) Chem.-Ztg. 1897, 790. 
ab. Die französische Pharmakopöe läßt als einzige die Menge des mit Hilfe von verdünnter Säure aus $0,5 \mathrm{~g}$ reduzierten Eisens entwickelten Wasserstoffes volumetrisch bestimmen und erzielt mit dieser Methode, wie $\mathrm{P} \mathrm{e} \mathbf{c ~ k}^{\mathbf{1}}$ ) gezeigt hat, wohl die einwandfreiesten Resultate. Dieses Verfahren ist jedoch ziemlich umständlich und erfordert die Beschaffung einer besonderen Apparatur. Dasselbe ist daher gegenüber einer mit den gebräuchlichen Gerätschaften ausführbaren Methode entschieden im Nachteil.

Auch die britische Pharmakopöe nimmt eine Sonderstellung unter den Arzneíbüchern betreffs der Gehaltsbestimmung des Ferrum reductum ein. Sie benutzt die Reduktionswirkung des metallischen Eisens auf Kupfersulfat und läßt das von dem abgeschiedenen Kupfer getrennte Filtrat mit volumetrischer Kaliumdichromatlösung titrieren. Dieses Verfahren liefert jedoch nach $\left.\mathrm{P} \mathrm{e} \mathrm{c} \mathrm{k} \mathrm{(l.} \mathrm{c.)})^{2}\right)$ zu hohe Werte.

Außer diesen Verfahren kommen - abgesehen von der eingangs besprochenen $\mathrm{W}$ in $\mathrm{k}$ le r'schen Methode des ungarischen Arzneibuches - im wesentlichen nur noch die von $\mathbf{E}$. S c h m i d t $\mathbf{t}^{\mathbf{3}}$ ) angegebene und von $A$. Marquard $t^{4}$ ) in etwas modifizierter Gestalt empfohlene Jodmethode und die auf der Reduktion des Quecksilberchlorids durch Metalle beruhende Quecksilberchloridmethode für die Bestimmung des metallischen Eisens im Ferrum reductum in Frage. Von diesen beiden Methoden benutzen zur Zeit nur noch wenige Arzneibücher (Oesterreich, Japan und Norwegen) die auf der Fähigkeit des Jods, metallisches Eisen zu lösen, beruhende Jodmethode. Dieses Verfahren lieferte solange gut übereinstimmende Werte ${ }^{5}$ ), als nur feinverteiltes und nicht zu hoch erhitztes, durch reines Wasserstoffgas reduziertes Eisen zur Verwendung gelangte. Durch den Wechsel in der Darstellungsmethode und die hierdurch veränderten Eigenschaften des Ferrum reductum hat jedoch die Jodmethode an Bedeutung verloren, zumal hierbei eine zweifache exakte Wägung (Eisen und Jod) erforderlich ist, und die tiefbraun gefärbte Flüssigkeit nur schwer erkennen läßt, ob Eisen und Jod vollkommen in Lösung gegangen sind.

1) Ebenda 1899 (23), 642.

2) Im Gegensatz zu Peck findet Frerich s (dieses Archiv 1908 (246), $190 \mathrm{ff}$.) nach der Kupfermethode annähernd genaue Werte.

3) Vers. deutscher Naturf. u. Aerzte 1897, Braunschweig (Chem.Ztg. 1897, 790).

4) Chem.-Ztg. 1901, 744.

5) Mündliche Mitteilung von Herrn Geheirrat E. Sch midt aus den Institutsjournalen der Jahre 1896-1898. 
Alle übrigen Arzneibücher lassen das metallische. Eisen durch Umsetzung mit Quecksilberchlorid bestimmen. Auch die amerikanische Pharmakopöe ist bei ihrer vorjährigen Neuausgabe von der Jod- zur Quecksilberchloridmethode übergegangen. Eine Zusammenstellung und eingehende'Besprechung der verschiedenen, wiederholt variierten Verfahren findet sich in der Arbeit von Frer i c h s${ }^{1}$, in welcher auch an Hand eines reichen Analysenmaterials nachgewiesen wird, daß auch die Quecksilberchloridmethode durchaus nicht in allen Fällen (trotz der von einzelnen Pharmakopöen vorgeschriebenen Vergrößerung der Menge des angewendeten Quecksilberchlorids und der Verlängerung der Einwirkungsdauer desselben bei Wasserbadtemperatur) befriedigende und übereinstimmende Werte liefert. Frerich s kommt daher zu dem Resultat, daß allein die bereits von $\mathrm{Parth} \mathrm{eil}^{2}$ ) empfohlene Bestimmung des Gesamteisengehaltes (gewichts- oder maßanalysisch) geeignet sei, den Wert des Ferrum reductum einwandfrei festzustellen, ,trotzdem die Berechnung des Gehaltes an metallischem Eisen aus dem Gesamteisengehalt nicht mit völliger Sicherheit ausgeführt werden kann". Das Verfahren besitzt nach Frerich s den weiteren Vorteil, daß die nicht als metallisches Eisen vorhandenen Bestandteile wenigstens keine fremden Verunreinigungen sein können, sondern aus Eisen in verschiedenen Oxydationsstufen bestehen.

Die Ausführungen Frer i c h' haben wohl auch dazu beigetragen, daß vom Deutschen Arzneibuch (5. Ausgabe) an Stelle der Bestimmung des metallischen Eisens diejenige des Gesamteisengehaltes aufgenommen wurde, nachdem sich sowohl die vom D. A.-B. III vorgeschriebene Quecksilberchloridmethode trotz ihrer Abänderung gelegentlich der Neuausgabe im Jahre 1895, als auch das durch die folgende (4.) Ausgabe des Deutschen Arzneibuches eingeführte Jodverfahren als nicht geeignet für alle Handelspräparate erwiesen hatten.

Wie zu erwarten war, hat dieser Uebergang von der Bestimmung des metallischen Eisens zu der des Gesamteisens bei dem Erscheinen der .5. Ausgabe des Deutschen Arzneibuches eine sehr verschiedenartige Beurteilung gefunden. Während einerseits Stimmen laut wurden ${ }^{3}$ ), die nur an ein versehentliches Fehlen der Bestimmung des metallischen Eisens glaubten, wurde von anderer Seite ${ }^{4}$ ) in An-

1) Dieses Archiv 1908 (246), $190 \mathrm{ff}$.

2) Apoth.-Ztg. 1890, 55 .

3) D üst er b eh n, Apoth.-Ztg. 1911, 185.

4) Cobl e ntz u. M a y, Ztschr. f. angew. Chem. 1909, I., 1224. 
lehnung an Frerich s' Arbeit der Einwand erhoben, daß wohl nur das feinverteilte Eisen im Organismus wirksam sein könne, während das Oxyduloxyd ebenso wie die gröberen Eisenpartikelchen den Verdauungsweg unverändert passierten und daß, da Jod und Quecksilberchlorid jedenfalls bezüglich des Lösungsvermögens für Eisen nicht mehr zu leisten vermöchten als die Verdauungssäfte, demnach eine Gehaltsbestimmung des metallischen Eisens unbedingt erforderlich sei.

Es scheint daher angezeigt zu sein, daß die kommende Neuausgabe des Deutschen Arzneibuches neben dem Gesamteisen auch das metallische Eisen besonders bestimmen läßt, um so mehr als die Berechnung des letzteren aus dem Gesamteisengehalt nur mit annähernder Genauigkeit möglich ist.

Um ein direktes Bild von dem Reaktionsverlauf der in den Arzneibüchern vorgeschriebenen Methoden zu gewinnen, wurde ein 97,6-97,85\% Gesamteisen enthaltendes Präparat nach den verschiedenen Verfahren unter Berücksichtigung der bekannt gewordenen Verbesserungsvorschläge untersucht, und zwar dienten für die Versuche einerseits die Vorschrift des D. A.-B. III, abgeändert durch den Neudruck 1895, andererseits die Angaben des D. A.-B. IV, variiert nach $M a r q u a r t^{1}$ ), und schließlich die Kupfermethode nach der Abänderung von $\mathrm{Coblentz}$ und $\mathrm{Ma}$ (l. c.). Letztere Autoren hatten hiermit einwandfreie, wohlübereinstimmende Resultate erhalten, während $\mathrm{P}$ e $\mathrm{k}^{2}$ ) wesentlich $\mathrm{zu}$ hohe Werte fand.

Quecksilberch lorid methode.

0,3 g Ferrum reductum wurden mit 5 g Quecksilberchlorid und $50 \mathrm{cem}$ Wasser eine Stunde lang auf dem Wasserbad erhitzt, das Gemisch wurde dann nach dem Erkalten auf $100 \mathrm{ccm}$ aufgefüllt, filtriert, und von dem Filtrat je $10 \mathrm{ccm}$ nach Zusatz von verdünnter Schwefelsằure mit frisch eingestellter Permanganatlösung titriert. Die austitrierte Lösung wurde darauf durch Weinsäure entfärbt, mit $2 \mathrm{~g}$ Jodkalium versetzt und nach einstündigem Stehen mit $1 / 10-N$.-Thiosulfatlösung titriert.

Permanganatverbrauch für $10 \mathrm{ccm}$ :

$$
\begin{gathered}
2,30 \mathrm{~cm}(1 \mathrm{cem}=0,009361 \mathrm{~g} \mathrm{Fe})=0,2153 \mathrm{~g} \text { Eisen. } \\
\text { Gefunden: } 7 \mathrm{~J}, 77 \% .
\end{gathered}
$$

1) Chem.-Ztg. 1901, 744.

2) Ebenda 1899, 642. 
Thiosulfatverbrauch für $10 \mathrm{ccm}$ :

$$
\begin{gathered}
9,44 \mathrm{ccm} 1 / 10-\mathrm{N}:-=0,21508 \mathrm{~g} \text { Eisen. } \\
\text { Gefunden: } 71,50 \% . \\
\text { Jod meth od e: }
\end{gathered}
$$

$0,3 \mathrm{~g}$ Ferrum reductum wurden mit $1 \mathrm{~g}$ Jodkalium, $4 \mathrm{~g}$ Wasser und 1,5127 $\mathrm{g}$ Jod in einem verschlossenen Fläschchen eine Stunde lang unter häufigem Umschütteln in Reaktion gebracht, das Gemisch wurde hierauf mit Wasser in einem $100 \mathrm{ccm}-K o l b e n$ gespült und zur Marke aufgefüllt. In $50 \mathrm{ccm}$ der Lösung wurde schließlich das überschüssige Jod bestimmt.

Thiosulfatverbrauch :

$12,46 \mathrm{ccm}=0,25824 \mathrm{~g} \mathrm{Jod}$ in $50 \mathrm{ccm}=0,5165 \mathrm{~g}$ Jod in der Gesamtrnenge $=0,9962 \mathrm{~g}$ durch Eisen gebundenes Jod $=0,2725 \mathrm{~g}$ metallisches Eisen.

$$
\text { Gefunden: 73,08\%. }
$$

Kupfersulfatmethode.

$0,3 \mathrm{~g}$ Ferrum reductum wurden mit $1,5 \mathrm{~g}$ Kupfersulfat und 15 com Wasser eine halbe Stunde auf dem Wasserbad erhitzt, das Gemisch wurde alsdann verschlossen beiseite gestellt und nach dem Erkalten auf $100 \mathrm{ccm}$ aufgefüllt. Von dem Filtrat wurden je $25 \mathrm{ccm}$ nach Zusatz von $20 \mathrm{ccm}$ Phosphorsäure von $25 \%$ mit eingestellter Permanganatlösung titriert.

1. Permanganatverbrauch für $25 \mathrm{ccm}$ Filtrat $=7,841 \mathrm{ccm}(1 \mathrm{ccm}=$ $0,009361 \mathrm{~g} \mathrm{Fe})=0,0734 \mathrm{~g} \mathrm{Fe}$;

für die Gésamtmenge $(0,3 \mathrm{~g})=0,2936 \mathrm{~g} \mathrm{Fe}=97,90 \%$.

2. Permanganatverbrauch für $25 \mathrm{ccm}$ Filtrat $=7,787 \mathrm{ccm}=$ $0,072894 \mathrm{~g} \mathrm{Fe}$;

für die Gesamtmenge $(0,3 \mathrm{~g})=0,29157 \mathrm{~g} \mathrm{Fe}=97,19 \%$.

Während also die beiden ersten Methoden wesentlich zu niedrige Resultate ergeben hatten, lieferte die Kupfermethode zu hohe Werte, wie bereits P e c k (1. c.) festgestellt hat. Da F r e r i c h s (l. c.) an Hand eines reichen Analysenmaterials die Unzulänglichkeit der einzelnen Verfahren und ihrer Variationen bewiesen hat, so glaubte ich, auf eine Wiederholung dieser Prüfungen verzichten und mich auf die Ausführung der Quecksilberchloridmethode in einer, in der Literatur mehrfach empfohlenen ${ }^{\mathbf{1}}$ ) Abänderung beschränken zu

1) Chem.-Ztg. 1901, 744; Apoth.-Ztg. 1908, 256. 
können. Nach der letzteren, von $\mathrm{M} \mathrm{e} \mathrm{c} \mathrm{k}^{1}$ ) ausgearbeiteten Methodewird in einem $100 \mathrm{ccm}$-Kolben $1 \mathrm{~g}$ Eisen mit $5 \mathrm{~g}$ Quecksilberchlorid und $50 \mathrm{ccm}$ Wasser auf dem Drahtnetz mit kleiner Flamme unter Umschwenken langsam zum Sieden erhitzt, das Gemisch dann noch eine Minute lang im Sieden erhalten, nach dem sofortigen Auffüllen bis zur Marke verschlossen erkalten gelassen und nunmehr definitiv auf $100 \mathrm{ccm}$ aufgefüllt. In je $10 \mathrm{ccm}$ der filtrierten Flüssigkeit wird nach Zusatz von $10 \mathrm{ccm}$ verdünnter Schwefelsäure schließlich der Eisengehalt mit 1/10-N.-Permanganatlösung bestimmt. Um hierbei eine Oxydation des Eisens durch den Luftsauerstoff möglichst zu vermeiden, wird die Hauptmenge der Permanganatlösung auf einmal zugesetzt und erst dann tropfenweise zu Ende titriert. Zur Kontrolle wird die Lösung noch mit $2 \mathrm{~g}$ Jodkalium versetzt und nach halbstündigem Stehen das ausgeschiedene Jod mit 1/10N.Thiosulfatlösung titriert.

Die nach diesem Verfahren durchgeführten Untersuchungen ergaben im Gegensat: zu den obigen Bestimmungen für das metallische Eisen wesentlich andere, jedoch unter sich übereinstimmende Werte, die dem aus dem Gesamteisen berechneten Eisengehalt sehr nahe kamen und, da letztere Berechnung nur angenäherte Resultate liefert, wohl dem wahren Eisengehalt entsprechen.

Bestimmung des Gesamteisens:

l. 2.

Permanganatverbrauch
$(1 \mathrm{ccm}=0,009361 \mathrm{~g} \mathrm{Fe}) . . .10,40 \mathrm{ccm}$
$=$ Prozentgehalt . . . . . 97,35\%
$10,50 \mathrm{ccm}$
$98,29 \%$
$10,33 \mathrm{ccm}$
Thiosulfatverbrauch . . . . . $17,46 \mathrm{~cm}$
$17,505 \mathrm{ccm}$
$96,64 \%$
$=$ Prozentgehalt . . . . . 97,60\%
$97,85 \%$
$17,47 \mathrm{com}$
$97,65 \%$

Berechnung desmetallischen Eisens:

$$
93,93 \% \quad 94,84 \% \quad 94,10 \%
$$

Bestimmung des metallisehen Eisens:

$$
\text { 1. } 2 . \quad 3 \text {. } 4 \text {. } 5 \text {. } 6 \text {. }
$$

Permanganatverbrauch in

Kubikzentimetern $\quad \ldots \quad \begin{array}{llllll}10,13 & 10,08 & 9,95 & 10,00 & 10,05 & 10,00\end{array}$

$\begin{array}{lllllll}\text { Prozentgehalt . . . . . } & 94,83 & 94,36 & 93,14 & 93,61 & 94,08 & 93,61\end{array}$

$\begin{array}{lllllll}\text { Thiosulfatverbrauch in cem } \quad 16,908 & 16,815 & 16,77 & 16,834 & 16,83 & 16,81\end{array}$

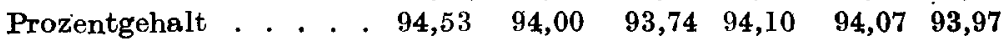

1) Merck's Jahresberichte 1900, 30.

(SchluB folgt.) 
Da die M e r c k'sche Bestimmungsmethode somit sehr brauchbare Resultate zu liefern schien, wurden zwei weitere, schon längere Zeit im hiesigen Institut gebrauchte Eisenproben derselben Behandlung unterzogen. Ein dem obigen Präparat sehr nahestehendes Ferrum reductum ergab folgende Werte:

Bestimmung des Gesamteisens:

1. 2 . 3 .

Permanganatverbrauch

$(1 \mathrm{ecm}=0,009361 \mathrm{~g} \mathrm{Fe}) \cdot .10,50 \mathrm{ccm} \quad 10,40 \mathrm{ccm} \quad 10,45 \mathrm{ccm}$ Prozentgehalt . . . . . . . 98,29\% $\quad 97,35 \% \quad 97,82 \%$ Thiosulfatverbrauch . . . . $17,47 \mathrm{ccm} 17,505 \mathrm{ccm} 17,56 \mathrm{ccm}$ Prozentgehalt . . . . . 97,65\% 97,85\% 98,16\%

Berechnung des metallischen Eisens:

$$
93,93 \% \quad 94,84 \% \quad 95,96 \%
$$

Bestimmung des metallischen Eisens:

1 .

Permanganatverbrauch . . . . 10,04 cem

Prozentgehalt . . . . . . 93,98\%

Thiosulfatverbrauch . . . . . 16,77 ccm

Prozentgehalt . . . . . . 93,74\%
2.

$9,93 \mathrm{ccm}$

$92,95 \%$

$16,72 \mathrm{ccm}$

$93,46 \%$

Ebenso gleichmäßig waren die Werte, die eine stark oxydhaltige Probe von Ferrum reductum lieferte:

Bestimmung des Gesamteisens:

1.

2.

Permanganatverbrauch

(1 $\mathrm{ccm}=0,00936 \mathrm{I} \mathrm{Fe}) . .10,10 \mathrm{ccm} \quad 10,30 \mathrm{ccm}$

Prozentgehalt . . . . 94,55\% 96,42\%

Thiosulfatverbrauch . . . . $17,00 \mathrm{ccm} \quad 17,14 \mathrm{ccm}$

Prozentgehalt . . . . . . . 95,03\% 95,81\%

Berechnung des metallischen Eisens:

$$
81,20 \% \quad 83,40 \%
$$

Bestimmung des metallischen Eisens:

Permanganatverbrauch . . . 9,10 ccm $9,30 \mathrm{ccm}$

Prozentgehalt . . . . . . 84,78\% $86,64 \%$

Thiosulfatverbrauch . . . . . 15,37 cem $15,42 \mathrm{ccm}$

Prozentgehalt . . . . 85,92\% 86,19\%

Bemerkenswert ist, da $B$ die $M$ e $\mathbf{r}$ c k'sche Methode mit Reagens. mengen arbeitet, die den theoretisch erforderlichen kaum entsprechen.

Aroh. d. Pharm. CoLV. Bda 6. Hoft. 
Auch hierüber hat Fre rich s (l. c.) bereits Untersuchungen angestellt und ist zu dem Resultat gekommen, daß zur Bestimmung des metallischen Eisens im Ferrum reductum auf $1 \mathrm{~g}$ des Präparates $5 \mathrm{~g}$ Quecksilberchlorid praktisch nicht ausreichen, vielmehr die Menge des Eisens zu der des Quecksilberchlorids mindestens im Verhältnis 1: 10 stehen sollte, da die sich abspielende Reaktion nicht ausschließlich nach der Gleichung:

$$
\mathrm{HgCl}_{2}+\mathrm{Fe}=\mathrm{FeCl}_{2}+\mathrm{Hg}
$$

verläuft, sondern auch nach der Formel

$$
2 \mathrm{HgCl}_{2}+\mathrm{Fe}=\mathrm{Hg}_{2} \mathrm{Cl}_{2}+\mathrm{FeCl}_{2} \text {. }
$$

Letztere Formel erfordert fast $10 \mathrm{~g}$ Quecksilberchlorid a uf $1 \mathrm{~g}$ Eisen. Daß das Fünffache der Eisenmenge an Quecksilberchlorid aber schon völlig ausreicht, ergibt sich aus dem Vergleich mic Versuchen, die ich mit der im neuen Arzneibuch der Vereinigten Staaten (1916) gegebenen Vorschrift anstellte. Die von dieser Pharmakopöe aufgenommene Methode schließt sich dem Merck'schen Verfahren eng an: $1 \mathrm{~g}$ Eisen wird mit $10 \mathrm{~g}$ Quecksilberchlorid und $50 \mathrm{ccm}$ heißem Wasser 5 Minuten lang im Sieden erhalten, das Gemisch nach dem Erkalten mit luftfreiem Wasser zu $100 \mathrm{ccm}$ aufgefüllt, und werden schlieBlich $20 \mathrm{ccm}$ des Filtrates unter Schwefelsäurezusatz mit Permanganat titriert. Die auf diese Weise gefundenen Werte stimmen praktisch mit den nach dem M e $\mathrm{c}$ k'schen Verfahren erhaltenen Resultaten überein.

Fer r u m r ductum I.

Amerikanisches Arzneibuch:

Permanganatverbrauch $(1 \mathrm{ccm}=0,009361 \mathrm{~g} \mathrm{Fe}) . \quad 9,948 \quad 9,985 \mathrm{ccm}$ Prozentgehalt . . . . . . . . . . . . . . . 93,12 93,47\%

M $\in$ r c k'sches Verfahren:

$\begin{array}{lrrrrrl}\text { Permanganatverbrauch } & 10,13 & 10,08 & 9,95 & 10,00 & 10,05 & 10,00 \mathrm{ccm} \\ \text { Prozentgehalt . . . . } & 94,83 & 94,36 & \mathbf{9 3 , 1 4} & 93,61 & \mathbf{9 4 , 0 8} & \mathbf{9 3 , 6 1 \%}\end{array}$

Ferrum reductum II.

Amerikanisches Arzneibuch:

Permanganatverbrauch . . . . . . . . . $9,227 \quad 9,256 \mathrm{ccm}$ Prozentgehalt . . . . . . . . . 86,37 86,65\%

M e r c k'sches Verfahren:

Permanganatverbrauch . . . . . . . . . 9,10 9,30 ccm Prozentgehalt . . . . . . . . . 84,78 86,64\% 
Hieraus ergibt sich, daß sowohl die Hälfte der von der amerikanischen Pharmakopöe vorgeschriebenen Quecksilberchloridmenge, als auch ein nur eine Minute dauerndes Sieden des Reaktionsgemisches genügt, um die Gesamtmenge des metallischen Eisens zu lösen.

Frerichs hat bei den verschiedenen Variationen der Quecksilberchloridmethode auch unter ähnlichen Versuchsbedingungen gearbeitet. Auch er hat eine Probe $(0,3 \mathrm{~g})$ mit der fünffachen Menge (1,5 g) Quecksilberchlorid und $15 \mathrm{ccm}$ Wasser 5 Minuten lang zum Sieden erhitzt, aber trotzdem keine einheitlichen Resultate erzielt. Die erhaltenen Analysenwerte schwankten zwischen 88,41\% und 94,80\%. Da die angewendeten Mengen der Merck'schen Vorschrift entsprechen ( $\mathrm{M}$ e r c k: $1 \mathrm{~g} \mathrm{Fe,} 5 \mathrm{~g} \mathrm{HgCl}, 50 \mathrm{ccm}$ Wasser); so kann der Mißerfolg der Frer i ch s'schen Versuche vielleicht dadurch bedingt sein, daß die Flüssigkeitsmenge (15 ccm) zu schnell zum Sieden kommt, oder daß durch das Umschwenken des Reaktionsgemisches in dem verhältnismäßig großen Kolben und durch das erstmalige Aufschäumen Eisenpartikelchen an den Gefäßwandungen abgelagert werden, die beim Sieden nicht wieder in die Flüssigkeit zurückgelangen und so dem Reaktionsbereich entzogen werden. Die von mir nach der M e r c k'schen Methode mehrfach durchgeführten. Analysen lieferten, wie aus vorstehenden Angaben hervorgeht, recht gut übereinstimmende Werte.

Eine weitere, den Angaben anderer Autoren nicht entsprechende, bei diesen Eisenbestimmungen gemachte Beobachtung ist die, daß bei der jodometrischen Kontrollbestimmung der mit Permanganat austitrierten Probe ein Zusatz von $2 \mathrm{~g}$ Jodkalium zur Jodabscheidung völlig ausreichend war, obwohl die Flüssigkeit überschüssig zugesetztes Quecksilberchlorid gelöst enthielt. Diese Beobachtung muß um so mehr befremden, als $\mathrm{W}$ e in $\mathrm{I}$ a $\mathrm{nd}^{\mathbf{1}}$ ) und in neuester Zeit F r e r i c h s${ }^{2}$ ) sogar bei der Bestimmung des Gesamteisengehaltes eine Erhöhung des Jodkaliumzusatzes von 2 auf $3 \mathrm{~g}$ empfehlen, obwohl sich hierbei nur Eisen und Schwefelsäure in dieser Lösung befinden. Durch direkte, unter Anwendung von noch größeren Mengen Jodkalium (3 bzw. $5 \mathrm{~g}$ ) angestellte Versuche konnte ich jedoch weder eine Erhöhung der ausgeschiedenen Jodmenge noch eine glattere Abwickelung der Reaktion feststellen. Bei der Bestimmung des metallischen bzw. Gesamteisens wurden zur Titration von je $10 \mathrm{ccm}$ der 1 : 100 bereiteten Lösung des hoch-

1) Maßanalyse (3. Aufl.), S. 106.

2) Apoth..Ztg. 1917, 203 u. 217. 
prozentigen Ferrum reductum zur Bindung des aus wechselnden Mengen Jodkalium freigemachten Jodes folgende Mengen Thiosulfatlösung verbraucht:

B tstimmung desmetallisehen Eisens:

Zuges. Menge Jodkalium $\quad 2 \mathrm{~g} \quad 3 \mathrm{~g} \quad \mathbf{5} \mathrm{g}$

Thiosulfatverbr. in ccm . $16,77 \quad 16,77 \quad 16,78 \quad 16,77 \quad 16,84 \quad 16,834$

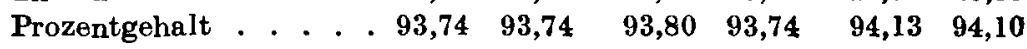

Bestimmung des Gesamteisens:

$\begin{array}{lll} & 2 \mathrm{~g} & 3 \mathrm{~g} \\ \text { Thiosulfatverbrauch in ccm . . . } & 17,56 & 17,57 \\ \text { Prozentgehalt . . . . . . } & 98,16 & 98,22\end{array}$

Ebensowenig konnte ich eine Beeinflussung der Werte durch Verkürzung der Einwirkungsdauer feststellen; nach halb- bzw. einstündigem Stehenlassen der Reaktionsflüssigkeiten ergaben sich praktisch stets dieselben Werte. Je $10 \mathrm{ccm}$ der $1: 100$ bereiteten Lösung desselben Eisenpräparates verbrauchten zur Titration des ausgeschiedenen Jodes folgende Mengen $1 / 10-\mathrm{N}$.-Thiosulfatlösung:

Bestimmung des metallischen Eisens: Einwirkungsdauer $1 / 2$ Stunde $\quad$ Stunde

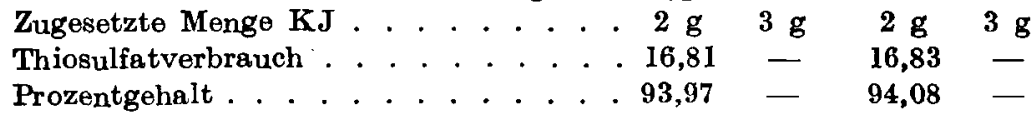

Bestimmung des Gesamteisens:

Thiosulfatverbrauch . . . . . . . . . . $17,474 \quad 17,48 \quad 17,47 \quad 17,51$ Prozentgehalt . . . . . . . . . . . 97,66 97,66 97,66 97,90

Die geringen Schwankungen in diesen Analysenbefunden sind praktisch ohne Bedeutung, da sie sich innerhalb der analytischen Fehlergrenzen bewegen. Ferner standen die Resultate dieser jodometrischen Bestimmungen stets im Einklang mit den auf oxydimetrischem Wege gefundenen Werten, besonders dann, wenn die von $\mathrm{Mer} \mathbf{k}$ angegebene Vorsichtsmaßregel beobachtet wurde, zunächst die Hauptmenge der Permanganatlösung auf einmal zuzusetzen und erst dann tropfenweise zu Ende zu titrieren. Infolgedessen möchte ich mich dem von Frerich s für die Bestimmung des Gesamteisengehaltes gemachten Vorschlag anschließen, die jodometrische Kontrolle entweder ganz fallen zu lassen (um so mehr als dadurch neben dem von Frerich s angeführten Zeitgewinn eine erhebliche Ersparnis an dem hoch im Preise stehenden Jodkalium ermöglicht wird, und eine Kontrolle des bei der ersten Titration gefundenen Wertes durch Wiederholung mit einem weiteren 
abgemessenen Quantum des Filtrates leicht und sicher bewerkstelligt werden kann), oder doch wenigstens für eine Gleichstellung der oxydimetrischen und jodometrischen Eisenbestimmung eintreten, so daß es in Zukunft in das Belieben des Analytikers gestellt wird, entweder die geringe Unbequemlichkeit der bei Bedarf vorzunehmenden einmaligen Neueinstellung der Permanganatlösung oder die Ausgaben für das zu jeder Bestimmung nötige Jodkalium in Kauf zu nehmen. Diesen Vorschlag möchte ich auch auf die nach der M e r c k'schen Vorschrift auszuführende Bestimmung des metallischen Eisens im Ferrum reductum ausdehnen.

Der einzige Uebelstand, der sowohl der Bestimmung des Gesamteisens als auch der des metallischen Eisens anhaftet, ist der, da $\beta$ das gefundene Resultat vertausendfacht werden muß, und da $\beta$ dadurch kleine Wäge- oder Ablesungsfehler erhebliche Differenzen in dem Gesamtresultat ergeben. Z. B. rief ein Mehr- oder Minderverbrauch von $0,1 \mathrm{ccm}$ der eingestellten Permanganatlösung $(1 \mathrm{ccm}=0,009361 \mathrm{~g} \mathrm{Fe})$ ein Schwanken des Eisengehaltes um $0,93 \%$ hervor. Indessen läßt sich diese Fehlerquelle einesteils durch die Anwendung einer verdünnteren Kaliumpermanganatlösung, anderenteils auch dadurch verringern, daß statt der bisher üblichen $10 \mathrm{ccm}$ Filtrat 20 oder $25 \mathrm{ccm}$ der Flüssigkeit angewendet werden, wie denn auch die neue Pharmakopöe der Vereinigten Staaten die Titration von $20 \mathrm{ccm}$ Filtrat vorschreibt. Dagegen ist die vom Schweizer Arzneibuch vorgeschriebene Verwendung von $50 \mathrm{ccm}$ der filtrierten Flüssigkeit zu verwerfen, da hierdurch die Fehlerquelle zwar stark verringert wird, aber eine Kontrolle mit derselben Lösung nur auf jodometrischem Wege möglich wäre, diese aber durch die großen Mengen Jodkalium, die zur Umsetzung nötig wären, unnötig verteuert würde. Würden dagegen zur Kontrolle $25 \mathrm{ccm}$ des Restfiltrates in diesem Falle verwendet, so würde dieses meinem Vorschlag entsprechen.

Neben den im vorstehenden dargelegten Bestimmungen wurde, im Anschluß an die Jodmethode, auf Anregung von Herrn Geheimrat E. Sch midt der Versuch gemacht, den Gehalt des Ferrum reductum an metallischem Eisen noch auf andere Weise zu ermitteln. Letzteren Versuchen lag die Erwägung zugrunde, daß die Reaktionsfähigkeit der Halogene in bezug auf das metallische Eisen mit abnehmendem Atomgewicht eine Steigerung erfährt, daß somit Brom wesentlich energischer auf metallisches Eisen einwirken muß als das höhermolekulare Jod. Der nach ein- bis mehrstündigem Digerieren durch das Eisen nicht gebundene Ueberschuß an Brom 
mußte sich durch Umsetzung mit Jodkalium und Titration des ausgeschiedenen Jods mit $1 / 10^{-\mathrm{N}}$.-Thiosulfatlösung dann bestimmen lassen. Unter Berücksichtigung der bei der Jodmethode gemachten Erfahrungen lag hierbei die Möglichkeit vor, die Gehaltsbestimmung des Ferrum reductum an metallischem Eisen noch bequemer, sls es nach der Quecksilberchloridmethode der Fall ist, mit Aussicht auf übereinstimmende Resultate zu gestalten.

Der direkten Anwendung des Broms stellte sich von vornherein die leichte Flüchtigkeit desselben als erhebliche Schwierigkeit in den Weg, die ein genaues Abwiegen des erforderlichen Halogens ausschloß. Diese Schwierigkeit ließ sich jedoch leicht durch indirekte, durch Wechselwirkung zwischen Kaliumbromat und Kaliumbromid in saurer Lösung bewirkte Bromabscheidung:

$$
\mathrm{KBrO}_{3}+5 \mathrm{KBr}+3 \mathrm{H}_{2} \mathrm{SO}_{4}=6 \mathrm{Br}+3 \mathrm{~K}_{2} \mathrm{SO}_{4}+3 \mathrm{H}_{2} \mathrm{O}
$$

umgehen, ähnlich wie es vom D. A.-B. 5 bereits für die Phenolbestimmung vorgeschrieben ist.

.Da einerseits Frerichs (l. c.) bei der Besprechung der Jodmethode gezeigt hat, daß die Reaktionsgeschwindigkeit der Halogene mit metallischem Eisen, entsprechend der Halogenkonzentration der Lösung, abnimmt, und andererseits die Verwendung größerer Flüssigkeitsmengen die Brauchbarkeit und leichte Durchführbarkeit der Methode beeinträchtigen mußte, so wurde statt der vom D. A.-B. 5 zur Phenolbestimmung vorgeschriebenen $1 / 100-\mathrm{N}$.-Kaliumbromatlösung eine Zehntel-Normallösung (16,702 g $\mathrm{KBrO}_{3}$ im Liter) angewandt, deren Wirkungswert durch blinde Versuche festgestellt wurde. Ferner wurde durch Verwendung entsprechender Mengen der Lösung $(50 \mathrm{ccm})$ für einen genügenden Bromüberschuß gesorgt, indem an Stelle von 1,4312 g Brom, welche zur Bindung von 0,5 g reinen Eisens erforderlich sind, 2,3976 $\mathrm{g}$ Brom,

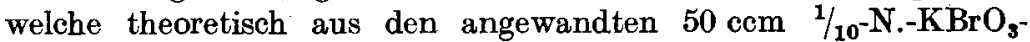
Lösung und entsprechenden Mengen Bromkalium freigemacht werden, zur Anwendung gelangten.

Das zur Einleitung der Reaktion notwendige Bromkalium wurde teils als $1 / 2$-Normallösung, teils, da ein Bromidüberschuß die Reaktion nicht beeinflußt, in Substanz zugesetzt. Es zeigte sich jedoch in letzterem Fall, daß die angewandte Flüssigkeitsmenge nicht genügend Brom in Lösung zu halten vermochte, so daß ein Teil des Broms, unterstützt durch die auftretende Reaktionswärme, den Stopfen der Glasstöpselflasche emporhob und teilweise entwich. Es wurde deshalb das erforderliche Bromkalium stets in Form der $1 / 2$-N.-Lösung angewandt. 
Die Versuche wurden in Rollflaschen von $300 \mathrm{ccm}$ Inhalt mit gut eingeschliffenem Glasstopfen durchgeführt, und zwar anfänglich. mit je $0,5 \mathrm{~g}$ Ferrum reductum (Gehalt an metallischem Eisen $=93,74-94,53 \%$, nach dem $M$ e r c k'schen Verfahren ermittelt) und je $50 \mathrm{ccm}$ Bromat- und Bromidlösung sowie $10 \mathrm{ccm}$ verdünnter Schwefelsäure $(1+5)$. Die Verwendung von Phosphorsäure an Stelle der Schwefelsäure erwies sich bei einem diesbezüglichen Versuch als ungeeignet (siehe unten). Da indessen die Menge des bromhaltigen Gemisches $(100 \mathrm{ccm})$ im Verein mit dem hohen Thiosulfatverbrauch (über $130 \mathrm{ccm}$ ) die Handhabung des Verfahrens erschwerte, wurden später nur $0,25 \mathrm{~g}$ Eisen mit je $25 \mathrm{ccm}$ der betr. Lösungen in Reaktion versetzt. Das analysierte Eisen.kam bei diesen Versuchen teils als Durchschnittsprobe ohne weitere Vorbehandlung, teils in feinzerriebenem Zustand zur Anwendung.

Zur Bestimmung des Wirkungswertes der Bromatlösung wurden gleichzeitig mit diesen Versuchen je $25 \mathrm{ccm}$ der. Lösung mit einer. solchen von Bromkalium und je $10 \mathrm{ccm}$ verdünnter Schwefelsäure $(1+5)$ zusammengebracht. Nach halbstündigem Stehen wurden dem Gemisch je $5 \mathrm{~g}$ Jodkalium zugegeben, und nach einer weiteren Stunde das ausgeschiedene Jod mit $1 / 10^{-N}$.Thiosulfatlösung zurücktitriert. Die bei diesen Bestimmungen gefundenen Werte zeigen jedoch so geringe Uebereinstimmung, da $B$ wohl angenommen werden mu $\beta$, da $\beta$ bei diesem Verfahren wechselnde Mengen Brom verloren gehen. Vor allem tritt dieser Uebelstand, dor bei einer späteren Einstellung einer neuen Kaliumbromatlösung vermieden werden konnte (siehe unten), bei dem Einbringen des Jodkaliums in die Rollflasche auf. Denselben Fehler müssen dementsprechend auch die mit dem Eisen angestellten Versuche selbst sufweisen. Für die Berechnung der Eisenbestimmungen wurde der höchste ermittelte Wert zugrunde gelegt.

Gehaltsbestimmung der Bromatlösung I.
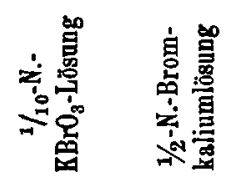

Jodkaliumzusatz

1. $25 \mathrm{ccm}$

$25 \mathrm{ccm}$

$5 \mathrm{~g}$ nach halbst. Stehen

2. $25 \mathrm{ccm}$

$25 \mathrm{ccm}$

58

3. $25 \mathrm{ccm}$

$25 \mathrm{ccm}$

5 " " "

$\begin{array}{rr}148,00 \mathrm{ccm} & 1,1828 \mathrm{~g} \\ 148,70 \mathrm{~cm} & 1,1884 \mathrm{~g} \\ 148,90 \mathrm{ccm} & 1,1900 \mathrm{~g} \\ =2,3800 \text { in } 50 \mathrm{ccm}\end{array}$

$\begin{array}{rr}148,00 \mathrm{ccm} & 1,1828 \mathrm{~g} \\ 148,70 \mathrm{~cm} & 1,1884 \mathrm{~g} \\ 148,90 \mathrm{~cm} & 1,1900 \mathrm{~g} \\ =2,3800 \text { in } 50 \mathrm{ccm}\end{array}$
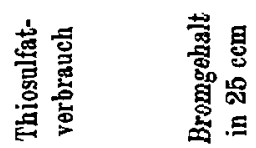

Theoretischer Wert: $1,1988 \mathrm{~g} \mathrm{Br}$ in $25 \mathrm{ccm}$ Lösung $=2,3976 \mathrm{~g} \mathrm{Br}$ in $50 \mathrm{ccm}$ Lösung 
Die erste Versuchsreihe galt der Erprobung der Brauchbarkeit dieser Methode überhaupt. Die Einwirkungszeit betrug bei den ersten drei Analysen je eine, bei Versuch 4 und 5 je 24 Stunden, wobei das Reaktionsgemisch anfangs längere Zeit ununterbrochen, später etwa alle 5 bis 10 Minuten umgeschwenkt wurde. Hierbei zeigte sich, daß die feineren Eisenpartikelchen sehr schnell (schon während der ersten 10 Minuten) in Lösung gingen, während die gröberen Anteile núr wenig verändert wurden. Nach ein- bzw. vierundzwanzigstündigem Stehen der Mischung wurden dann je $3 \mathrm{~g}$ Jodkalium zugesetzt und nach obigen Angaben weiter verfahren.

Versuch 1. 0,5000 g Eisen wurden mit $50 \mathrm{ccm} 1 / 10-\mathrm{N} .-\mathrm{KBrO}_{3}-$ Lösung, $50 \mathrm{ccm} \mathrm{1/2-N.-KBr-Lösung} \mathrm{und} 10 \mathrm{ccm}$ verdünnter Schwefelsäure $(1+5)$ zusammengebracht. Das Gemisch wurde zunächst durch Wasser gekühlt, später bei gewöhnlicher Temperatur stehen gelassen.

Thiosulfatverbrauch $133,46 \mathrm{ccm}=1,0666 \mathrm{~g}$ Brom.

Mithin gebunden an $\mathrm{Fe} 1,3134 \mathrm{~g} \mathrm{Br}=0,45884 \mathrm{~g}$ Eisen.

Gefunden: $91,77 \%$ metallisches Eisen.

Versuch 2. Ebenso wie 1, jedoch unter Anwendung von $0,5 \mathrm{~g}$ des obigen, vorher aber im Stahlmörser zerriebenen Eisens.

Thiosulfatverbrauch $135,45 \mathrm{ccm}=1,0825 \mathrm{~g}$ Brom.

Mithin gebunden an $\mathrm{Fe} 1,2975 \mathrm{~g} \mathrm{Br}=0,4533 \mathrm{~g}$ Eisen. Gefunden $90,60 \%$ metallisches Eisen.

Versuch 3. 0,5000 g $\mathrm{n} \mathrm{i} \mathrm{ch} \mathrm{t}$ zerriebenes Eisen wurden mik je $50 \mathrm{ccm}$ Bromat- und Bromidlösung und $20 \mathrm{ccm}$ offizineller Phosphorsäure von $25 \%$ versetzt. Nach kurzer Zeit wurde eine Trübung der anfangs klaren Flüssigkeit beobachtet, die sich zusehends verstärkte und schließlich zur Bildung einer dicken Gallerte führte. Die Titration des Gemisches erwies sich als undurchführbar, da ständig Nachbläuung eintrat (Thiosulfatverbrauch bis zur ersten Entfärbung nur $48,46 \mathrm{ccm}$ ).

Versuch 4. $0,5015 \mathrm{~g}$ fein $\mathrm{z}$ e $\mathrm{r} \mathrm{r}$ i e b e nes Eisen wurden wie bei Versuch 1 behandelt, die Einwirkungsdauer jedoch auf 24 Stunden a usgedehnt.

Thiosulfatverbrauch $132,40 \mathrm{ccm}=1,0581 \mathrm{~g}$ Brom.

Mithin gebunden an $\mathrm{Fe} 1,32 \mathrm{lg} \mathrm{Br}=0,4618 \mathrm{~g}$ Eisen.

Gefunden $92,08 \%$ metallisches Eisen.

Versuch 5. 0,5025 $\mathrm{g} \mathrm{n} \mathrm{i} \mathrm{cht} \mathrm{zerriebenes} \mathrm{Eisen} \mathrm{wurden} \mathrm{wie}$ bei Versuch 1 behandelt. 
Thiosulfatverbrauch $133,20 \mathrm{ccm}=1,0645 \mathrm{~g}$ Brom.

Mithin gebunden an $\mathrm{Fe} 1,3155 \mathrm{~g} \mathrm{Br}=0,4596 \mathrm{~g}$ Eisen.

Gefunden 91,46\% metallisches Eisen.

Dieser letzte Wert stimmt mit dem in Versuch 1 erhaltenen Resultat gut überein und zeigt somit, daß die Reaktion zwischen Eisen und Brom in der Hauptsache nach einstündigem Stehen beendet ist. Ebenso ist es einleuchtend, daßB durch Zerreiben im Stahlmörser eine feinere Verteilung und damit eine Erhöhung und Beschleunigung der Löslichkeit des Eisens erzielt wird, wie sie in dem Resultat von Versuch 4 zum Ausdruck kommt. Dieser Folgerung steht allerdings der in Versuch 2 erhaltene Analysenwert entgegen, der für das zerriebene Eisen einen niedrigeren Prozentgehalt aufweist als für nicht vorbehandeltes Material. In diesem Falle hatte also scheinbar die einstündige Einwirkungsdauer nicht zur völligen Auflösung des metallischen Eisens genügt.

Aus diesen einander widersprechenden Resultaten ergab sich die Notwendigkeit, die Beeinflussung der Analysenwerte durch verschieden lange Einwirkung von Brom auf reduziertes Eisen nachzuprüfen. $\mathrm{Zu}$ diesem $\mathrm{Zweck}$ wurden je $0,25 \mathrm{~g}$ reduziertes Eisen in $\mathrm{n}$ icht zerriebenem Zustand mit je $25 \mathrm{ccm} 1 / \mathrm{I}_{0}-\mathrm{N}$.-Bromat- und $1 / 2-\mathrm{N}$.-Bromidlösung sowie $10 \mathrm{ccm}$ verdünnter Schwefelsäure $(1+5)$ wie bei obiger Versuchsreihe zusammengebracht, nach 1, 2, 3, 6, 24 und 48 Stunden je $3 \mathrm{~g}$ Jodkalium zugesetzt und schließlich das nach einstündigem Stehen vorhandene freie Jod titriert.

\begin{tabular}{|c|c|c|c|c|c|c|c|}
\hline 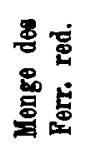 & & & 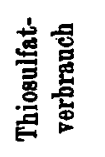 & 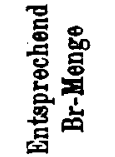 & 总量. & $\stackrel{\infty}{\square}$ & 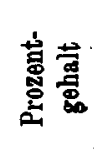 \\
\hline $\mathbf{g}$ & & & $\mathrm{ccm}$ & $\mathrm{g}$ & $g$ & g & $\%$ \\
\hline 0,2501 & & tunde & 68,37 & 0,5464 & 0,6436 & 0,22484 & 89,90 \\
\hline 0,2500 & & tunden & 68,54 & 0,5478 & 0,6422 & 0,22435 & 89,74 \\
\hline 0,2499 & 3 & ", & 66,95 & 0,5351 & $€, 6549$ & 0,2288 & 91,55 \\
\hline 0,2501 & 6 & , & 67,96 & 0,5431 & 0,6469 & 0,2260 & 90,36 \\
\hline 0,2501 & 24 & & 69,535 & 0,5557 & 0,6343 & 0,2216 & 88,60 \\
\hline 0,2502 & 48 & 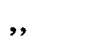 & 67,68 & 0,5417 & 0,6483 & 0,2265 & 90,52 \\
\hline
\end{tabular}

Die übereinstimmenden Werte der Versuche 1 und 2 zeigen, daß die Verlängerung der einstïndigen Einwirkungsdauer um eine weitere Stunde die Analysenresultate nicht zu beeinflussen vermag. In Gegensatz hierzu stehen die Ergebnisse der weiteren Versuehe 3 
bis 6, die von ersteren und untereinander starke Abweichungen (maximal um fast 3\%) zeigen. Zwar schließen sich die Versuchswerte 4 und 6 den Resultaten der Analysen 1 und 2 eng an (Differenz 0,46 bzw. 0,62\%). Dagegen stellen - die gleichmäßige Beschaffenheit des Analysenmaterials vorausgesetzt - die Resultate von 3 und 5 (Maximal- und Minimalwert) die Anwendbarkeit der Methode überhaupt in Frage. Immerhin aber konnten diese Werte durch eine von den anderen Proben abweichendé Beschaffenheit des Eisenpräparates (Anwesenheit größerer Partikelchen) bedingt sein, falls die Differenz nicht durch Bromverlust entstanden war. Deshalb wurde zu einer neuen Versuchsreihe das bei den ersten Versuchen übriggebliebene fein zerriebene Eisen verwendet, das in der Zwischenzeit (etwa 8 Tage lang) in einem Wägegläschen aufbewahrt worden war.

Da außerdem die erhaltenen Werte nicht mit den nach der M e r c k'schen Methode erzielten Resultaten $(93,74-94,53 \%$ metallisches Eisen) in Einklang standen, und die Einstellung der Bromatlösung I ergeben hatte, daß ihr Wirkungswert den berechneten Bromgehalt nicht erreichte, so wurde (um eine eventuell hieraus entstehende Fehlerquelle zu vermeiden) für die folgenden Versuche eine neue Bromatlösung hergestellt, deren Wirkungswert ebenfalls ermittelt wurde. Hierbei ergab sich, daß trotz des bei $100^{\circ}$ vorgenommenen Trocknens des Bromates die Wirkung der Lösung nicht dem theoretischen Wert entsprach, sich vielmehr den bei der Einstellung der ersten Kaliumbromatlösung erhaltenen Resultaten an die Seite stellte.

Gehaltsbestimmung der Bromat lösung II.

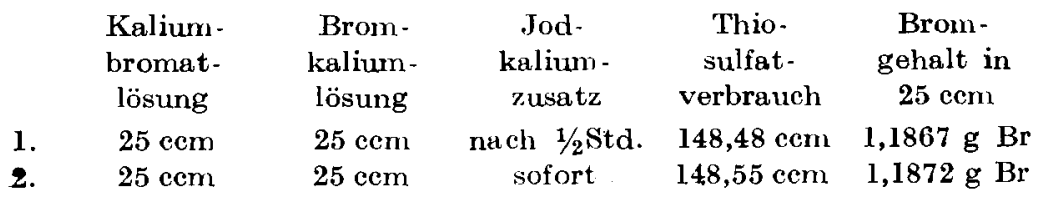

Da-auch bei sofortigem Jodkaliumzusatz keine höheren Werte gefunden wurden, mußte angenommen werden, daß schon bei dem Zusatz des Jodkaliums Bromverluste eintraten. Um diese zu vermeiden, wurde bei einem letzten Versuch das Jodkalium schon vor der Schwefelsäure der Lösung zugesetzt, bevor also Brom überhaupt in Freiheit gesetzt worden war. Durch diese Manipulation stieg der Thiosulfatverbrauch und damit der Wirkungswert der Lösung um ein Geringes an. 
$25 \mathrm{ccm}$ Bromatlösung verbrauchten nunmehr $149,05 \mathrm{ccm}=$ $1,1912 \mathrm{~g}$ Brom.

Wenn auch in diesem Fall der theoretische Wert $(1,1988 \mathrm{~g}$ Brom in $25 \mathrm{ccm}$ ) nicht erreicht wurde, so muß diese Differenz in einer Verunreinigung des Kaliumbromates gesucht werden.

Unter Zugrundelegung des so erhaltenen Wirkungswertes (1,1912 $\mathrm{g}$ Brom in $25 \mathrm{ccm}$ Bromatlösung) wurden nunmehr je 0,25 $\mathrm{g}$ Eisen wie bei den obigen Versuchen analysiert. Versuch 1 illustriert die Einwirkung des Jodes auf Eisen, die sich als wesentlich schwächer als die des Broms erweist.

\begin{tabular}{|c|c|c|c|c|c|c|}
\hline 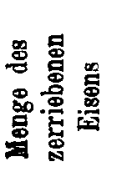 & 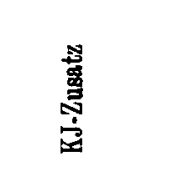 & 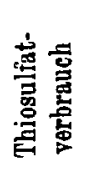 & 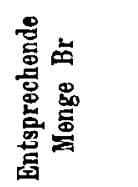 & 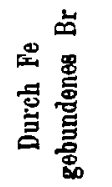 & 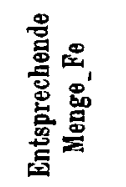 & 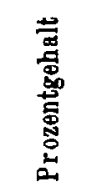 \\
\hline$\dot{\mathbf{g}}$ & & $\mathrm{ccm}$ & g & $\mathrm{g}$ & g & $\%$ \\
\hline 0,2508 & sofort & 81,37 & 0,6503 & 0,5409 & 0,1890 & 75,34 \\
\hline 0,2496 & nach I Std. & 74,15 & 0,592607 & 0,5986 & 0,20912 & 83,7 \\
\hline 0,2512 &,$\quad 2$, & 73,45 & 0,5870 & 0,6042 & 0,2111 & 84.03 \\
\hline 2509 & 3, & 74,80 & 0,5978 & 0,5934 & 0,2073 & 82,63 \\
\hline 502 &, 6, & 71,41 & 0,57071 & 0,6205 & 0,2168 & 86,64 \\
\hline 502 &, 24, & 70,47 & 0,5632 & 0,6280 & 0,2194 & 87,69 \\
\hline
\end{tabular}

Mit Ausnahme von Versuch 4, der völlig aus der Reihe fällt, zeigen die Resultate ein allmähliches Anwachsen mit steigender Einwirkungsdauer. Indessen sind die nach sechs-, noch weniger natürlich die nach 24stündigem Stehen des Gemisches erhaltenen Werte nicht mehr als zuverlässig anzusehen, da geringe Undichtigkeiten im Schliff der Glasstopfen einen Bromverlust nie ganz aus:schließen und somit einen erhöhten Eisengehalt vorzutäuschen imstande sind. Die Versuche würden also gezeigt haben, daß die Hauptmenge des Eisens nach einstündigem Stehen gelöst ist, und würden somit die Brauchbarkeit der Methode erwiesen haben, w e $\mathbf{n} \mathbf{n}$ die so erbaltenen Werte mit den Resultaten der nach anderen Methoden durchgeführten Bestimmungen in Einklang ständen. Dies ist aber nicht der Fall. Die trotz des Zerreibens des Eisens auffallend niedrigen Werte der letzten Versuchsreihe stimmen bei weitem nicht mit den Resultaten der Me r c k'schen Methode, die für das $n$ i c h t zerriebene Eisen bereits $93,74-94,53 \%$ metallisches Eisen ergeben hatte, überein. Daher wurde zur Kontrolle nunmehr mit dem Rest des obigen zerriebenen Analysenmateriales eine 
Bestimmung nach Merck ausgeführt, die diesmal auch einen wesentlich niedrigeren Wert als früher lieferte.

20 com der 1 : 100 bereiteten Lösung von 1,0004 g Ferr. red. verbrauchten $18,95 \mathrm{ccm}$ Permanganatlösung ( $1 \mathrm{ccm}=0,009455 \mathrm{~g} \mathrm{Fe}$ ) $=89,55 \%$ metallisches Eisen.

$20 \mathrm{ccm}$ der 1 : 100 bereiteten Lösung von 1,0004 g Ferr. red. verbrauchten $32,04 \mathrm{ccm} 1 / 10-\mathrm{N}$.-Thiosulfatlösung $=89,43 \%$ metallisches Eisen.

Ein Vergleich dieses Resultates mit den früher erhaltenen Werten $(93,74-94,53 \%)$ lehrt, daß das zerriebene Ferrum reductum bei dem Aufbewahren eine Oxydation erfahren haben muß. Gleichzeitig lassen diese Analysenergebnisse aber auch erkennen, daß die nach dem Bromverfahren erhaltenen Werte um mehrere Prozente von den Resultaten der M e r c k'schen Methode differieren.

Zur Bestätigung dieser Beobachtung wurde schließlich noch eine Probe des nicht zerriebenen Eisens einer vergleichenden Untersuchung nach dem M er ck'schen und dem Bromverfahren unterzogen.

Merck'sches Verfahren:

$20 \mathrm{cem}$ der Lösung von $1,0010 \mathrm{~g}$ Ferr. red. in $100 \mathrm{ccm}$ ver brauchten oxydimetrisch: 19,70 ccm Permanganat $=93,04 \% \mathrm{Fe}$, jodometrisch: $33,32 \mathrm{ccm} 1 / 10-N$. Thiosulfat $=92,94 \%$ Fe.

Brommethode:

\begin{tabular}{|c|c|c|c|c|c|}
\hline $\begin{array}{l}\text { Yenge } \\
\text { des Ferrum } \\
\text { reductum }\end{array}$ & $\begin{array}{l}\text { Ein- } \\
\text { wirkangs- } \\
\text { dauer }\end{array}$ & $\begin{array}{l}\text { Thiosulfat- } \\
\text { verbrauch }\end{array}$ & $\begin{array}{c}\text { Ent- } \\
\text { sprechende } \\
\text { Menge Br }\end{array}$ & $\begin{array}{c}\text { Metallisehes } \\
\text { Eisen }\end{array}$ & $\begin{array}{l}\text { Prozent- } \\
\text { gehalt }\end{array}$ \\
\hline 0,25 & 2Stu & 73,2 & $0,5855 \mathrm{~g}$ & 0,21 & \\
\hline $0,2507 \mathrm{~g}$ & 15 & $67,23 \mathrm{ccm}$ & $0,5373 \mathrm{~g}$ & $0,2284 \mathrm{~g}$ & $91,12 \%$ \\
\hline
\end{tabular}

Auch hierbei zeigen die Resultate erhebliche Differenzen, wenigstens unter Heranziehung des nach zweistündiger Einwirkung des Bromat-Bromidgemisches erhaltenen Wertes. Alle durch längeres Stehenlassen erhaltenen Resultate leiden an einer gewissen Unsicherheit. Diese beruht, wie oben erwähnt, auf der leichten Flüchtigkeit des Broms, das schon bei ganz minimalen Undichtigkeiten entweicht, und wird durch den Umstand erhöht, daß ein Verlust an Halogen einen erhöhten Eisengehalt vortäuscht. Diese Schwierigkeit ließ sich zwar bei der Einstellung des Wirkungswertes der Bromatlösung vermeiden, nicht jedoch bei den Versuchen selbst, bei denen das Einbringen des Jodkaliums stets mit einem geringen 
Verlust an Bromdampf verbunden war. Die trotz dieses Umstandes und der dadurch bedingten Erhöhung der Resultate noch immer wesentlich zu niedrigen Werte sind wohl, wie bei der Jodmethode. auf eine zu geringe Konzentration der Bromlösung zurückzuführen. Da dieser Fehler sich aber, wie die Versuche unter Anwendung von Bromkalium in Substanz gezeigt haben, nicht vermeiden lieB, so mußte diese an sich sehr einfache Methode als ungeeignet aufgegeben werden.

Diese Versuche haben weiter gezeigt, daß das Bromverf hren bezüglich der Genauigkeit und GleichmäBigkeit der erzielten Resultate nicht wesentlich mehr zu leisten vermag als die Jodmethode, sodaß dieselbe der M e r c k'schen Quecksilberchloridmethode, die an Genauigkeit und Uebereinstimmung der Resultat nichts zu wünschen übrig läßt, sehr nachsteht.

In Zusammenfassung der Beobachtungen, welche ich bei den im vorstehenden beschriebenen Versuchen gemacht habe, gehen meine Vorschläge bezüglich der Wertbestimmung des Ferrum reductum darauf hinaus:

1. Bestimmung des metallischen Eisens nach der $\mathbf{M}$ e r c kschen Quecksilberchloridmethode neben der bisherigen Bestimmung des Gesamteisens,

2. Ausschaltung der jodometrischen Bestimmung sowohl bei der Ermittelung des Gesamteisengehaltes als auch bei der Kontrolle der oxydimetrischen Bestimmung des metallischen Eisens nach M e r c k, oder wenigstens Gleichstellung der oxydimetrischen Bestimmungsmethode und

3. Verwendung von je $25 \mathrm{ccm}$ des Filtrates (bei der Merck'schen Methode und der Gesamteisenbestimmung) zu jeder Bestimmung, gleichgültig, ob dieselbe auf oxydimetrischem oder auf jodometrischem Wege zur Ausführung gelangt. Bei den oxydimetrischen Bestimmungen empfiehlt ès sich, eine verdünnte Kaliumpermanganatlösung $\left(2-2,5 \mathrm{~g} \mathrm{KMnO}_{\text {\& }} 1000 \mathrm{ccm}\right)$ zur Titration zu verwenden. 